Fabian David Backx

\title{
Estimação de Canal e Detecção Cega de Sinais em Sistemas de Transmissão OFDM
}

Tese apresentada ao Programa de Pós-graduação em Engenharia Elétrica do Departamento de Engenharia Elétrica da PUC-Rio como requisito parcial para obtenção do título de Doutor em Engenharia Elétrica

Orientador: Dr. Raimundo Sampaio Neto 


\section{Fabian David Backx}

\section{Estimação de Canal e Detecção Cega de Sinais em Sistemas de Transmissão OFDM}

Tese apresentada como requisito parcial para obtenção do grau de Doutor pelo Programa de Pós-Graduação em Engenharia Elétrica do Departamento de Engenharia Elétrica do Centro Técnico Científico da PUC-Rio.

Aprovada pela Comissão Examinadora abaixo assinada.

Dr. Raimundo Sampaio Neto

Orientador

Centro de Estudos em Telecomunicações/PUC-Rio

Dr. Ernesto Leite Pinto

IME

Dr. Marcello Luiz Rodrigues de Campos

COPPE/UFRJ

Dr. José Mauro Pedro Fortes

Centro de Estudos em Telecomunicações/PUC-Rio

Dr. Weiler Alves Finamore

Centro de Estudos em Telecomunicações/PUC-Rio

Dr. Sergio Lima Netto

COPPE/UFRJ

Prof. José Eugenio Leal

Coordenador Setorial do Centro Técnico Científico -

PUC-Rio

Rio de Janeiro, 03 de Abril de 2009 
Todos os direitos reservados. É proibida a reprodução total ou parcial do trabalho sem autorização da universidade, do autor e do orientador.

Fabian David Backx

Graduou-se em Engenharia de Telecomunicações na UFF (Universidade Federal Fluminense) em 2002. Recebeu o título de Mestre em Engenharia Elétrica na área de Sistemas de Comunicação pela PUC-Rio em 2004.

Ficha Catalográfica

David Backx, Fabian

Estimação de Canal e Detecção Cega de Sinais em Sistemas de Transmissão OFDM / Fabian David Backx; orientador: Raimundo Sampaio Neto . - 2009.

119 f. : il. (color.) ; $30 \mathrm{~cm}$

Tese (Doutorado em Engenharia Elétrica) - Pontifícia Universidade Católica do Rio de Janeiro, Rio de Janeiro, 2009.

Inclui bibliografia

1. Engenharia Elétrica - Teses. 2. OFDM. 3. Estimação cega de canal. 4. Estimação de canal via Subespaço de ruído. 5. Casamento de correlação. I. Sampaio Neto, Raimundo. II. Pontifícia Universidade Católica do Rio de Janeiro. Departamento de Engenharia Elétrica. III. Título. 


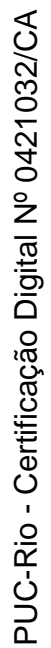

Aos meus pais, Arago e Janine. 


\section{Agradecimentos}

Ao professor Raimundo, pela orientação e ajuda na elaboração deste trabalho. Muito obrigado por todos os seus ensinamentos, e por estar sempre disposto a ingressar nos mais variados debates suscitados pelas minhas eternas dúvidas.

Aos membros da banca examinadora, pelas valiosas sugestões que contribuíram para enriquecer a versão final deste texto.

À minha família, por todo o carinho e suporte, e pela confiança em mim depositada.

Aos meus amigos. Em especial aos meus colegas do laboratório de Sistemas de Comunicação ao longo de todos estes anos: Amanda Cunha, Bernardo Costa, Carol Finamore, Cesar Medina, Cláudia Quevedo, Danilo Silva, Deolinda Cardoso, Fred Berkowicz, José Antônio Seiblitz, Juan Otálora, Marcio A. de Souza, Rodrigo de Lamare, e Tiago Vinhoza.

Ao CNPq e à PUC-Rio, pelo apoio financeiro concedido. 


\section{Resumo}

David Backx, Fabian; Sampaio Neto, Raimundo (Orientador) . Estimação de Canal e Detecção Cega de Sinais em Sistemas de Transmissão OFDM. Rio de Janeiro, 2009. 119p. Tese de Doutorado — Departamento de Engenharia Elétrica, Pontifícia Universidade Católica do Rio de Janeiro.

O esquema OFDM (Orthogonal Frequency Division Multiplexing) é um esquema de transmissão de sinais por multiplexação em freqüência e sua adoção vem mostrando-se bastante promissora no que diz respeito à transmissão de sinais em canais seletivos em freqüência. Sua relativa robustez frente a canais com múltiplos percursos é conseguida efetuando-se a transmissão paralela de dados em subportadoras ortogonais estreitas. Os receptores OFDM, de uma forma geral, necessitam de estimativas do canal de comunicação para realizar a detecção coerente dos sinais recebidos. Estimativas do canal podem ser obtidas de forma assistida por meio da utilização de pilotos que consomem preciosa banda, ou de forma cega, valendo-se apenas do conhecimento de características estatísticas dos sinais transmitidos. Esta tese segue a linha de estimação cega de canal. São propostos dois estimadores cegos de canal, para sistemas OFDM com intervalo de guarda $\mathrm{ZP}$ ou $\mathrm{CP}$. O primeiro baseia-se no casamento de correlação, a saber: a estimativa do canal corresponde ao argumento que minimiza a norma quadrática de Frobenius da diferença entre a matriz correlação teórica parametrizada dos sinais recebidos e uma estimativa desta matriz obtida por meio das observações no receptor. O segundo estimador é desenvolvido a partir de uma modificação do método de identificação de canal por meio da estimação do subespaço de ruído, lançando mão de uma estimativa para o operador projeção no subespaço de ruído. Técnicas para resolver a ambigüidade complexa presente nas estimativas do canal que resultam dos métodos propostos são abordadas. Os estimadores propostos são estendidos para sistemas OFDM ditos com intervalo de guarda insuficiente. Resultados de simulações ilustram o desempenho, tanto em termos de erro quadrático médio dos estimadores, quanto em taxa de erro de bit dos sistemas.

\section{Palavras-chave}

OFDM. Estimação cega de canal. Estimação de canal via Subespaço de ruído. Casamento de correlação. 


\section{Abstract}

David Backx, Fabian; Sampaio Neto, Raimundo (Advisor) . Blind Channel Estimation and Signal Detection in OFDM Transmission Systems. Rio de Janeiro, 2009. 119p. DSc Thesis — Departmento de Engenharia Elétrica, Pontifícia Universidade Católica do Rio de Janeiro.

Orthogonal Frequency Division Multiplexing is a multi-carrier transmission technique suitable for wireless communications through frequency selective channels, thus making it an appealing scheme for next-generation applications demanding high data rates. Its robustness with respect to multipath channels is obtained by modulating a set of closely-spaced orthogonal sub-carriers. In order to coherently detect the received signals, Channel State Information must be available to OFDM receivers. Supervised channel estimation is achieved by multiplexing known pilot symbols and data symbols, thus reducing effective system throughput. On the other hand, unsupervised or blind estimation techniques rely solely on the knowledge of statistical characteristics of the transmitted signal in order to identify the channel. This thesis proposes two blind channel estimators, for OFDM systems with CP or ZP guard interval. The first estimator is based on correlation matching: a channel estimate is obtained as the argument which minimizes the Frobenius quadratic norm of the difference between the parameterized theoretical correlation matrix of the transmitted signals and an estimate of that matrix obtained by means of observations at the receiver. The second estimator is obtained by modifying the noise subspace based channel estimator and using an estimate for the noise subspace projector which relies on powers of the inverse correlation matrix. Techniques to eliminate the inherent complex ambiguity derived from the proposed blind channel estimators are addressed. The porposed estimators' formulation is also extended to the case of OFDM systems with insufficient guard intervals. Simulation results depict Mean Square Error of the proposed estimators, as well as Bit Error Rate performance of systems using those estimators in various scenarios.

\section{Keywords}

OFDM. Blind channel estimation. Noise subspace based channel estimation. Correlation matching. 


\section{Sumário}

1 Introdução $\quad 13$

1.1 Breve Histórico do OFDM 13

$\begin{array}{ll}1.2 \text { Motivação e Objetivo } & 14\end{array}$

1.3 Organização do Texto 17

$\begin{array}{ll}1.4 \text { Notação Adotada } & 18\end{array}$

2 Modelo de Sinais $\quad 19$

$\begin{array}{ll}2.1 \text { Introdução } & 19\end{array}$

2.2 Modelo de Comunicação 19

2.2.1 Transmissão do Sinal OFDM 19

2.2.2 Recepção do Sinal OFDM 22

3 Receptores OFDM para Intervalo de Guarda Suficiente 27

$\begin{array}{lll}3.1 & \text { Introdução } & 27\end{array}$

$\begin{array}{ll}3.2 \text { Receptores Convencionais } & 27\end{array}$

3.2.1 Receptor Convencional para OFDM-CP 27

3.2.2 Receptor Convencional para OFDM-ZP 30

3.3 Analogia entre os Sinais OFDM e CDMA 36

4 Estimação Cega de Canal para Intervalo de Guarda Suficiente 38

4.1 Introdução 38

4.2 Estimação Cega de Canal por meio do Subespaço de Ruído 38

4.3 Método das Potências: OFDM-ZP 42

4.3.1 Implementação Alternativa pós-DFT 45

4.4 Método das Potências: OFDM-CP 49

4.5 Casamento de Correlação: OFDM-ZP 53

4.6 Casamento de Correlação: OFDM-CP 57

$\begin{array}{lll}4.7 & \text { Resultados } & 59\end{array}$

4.7.1 Preliminares $\quad 59$

4.7.2 Casamento de Correlação - OFDM-ZP 59

4.7.3 Método das Potências - OFDM-ZP 66

4.7.4 Casamento de Correlação - OFDM-CP 72

4.7.5 Método das Potências - OFDM-CP 75

4.7.6 Estimação de $\sigma^{2}$ - OFDM-ZP

4.7.7 Expressão Analítica do MSE - OFDM-ZP 80

4.8 Resolvendo a Ambigüidade - OFDM-ZP 83

5 Sistemas OFDM com Intervalo de Guarda Insuficiente $\quad \mathbf{8 8}$

$\begin{array}{lll}5.1 & \text { Introdução } & 88\end{array}$

5.2 Detecção de Sinais 88

5.3 Estimação Cega de Canal 88

5.3.1 Estimação de Canal por meio do Método das Potências 89

5.3.2 Estimação de Canal com Casamento de Correlação 97

$\begin{array}{lll}\text { 5.3.3 Resultados } & 100\end{array}$ 
$\begin{array}{lll}6 & \text { Conclusão } & 104\end{array}$

$\begin{array}{ll}\text { Referências Bibliográficas } & 107\end{array}$

$\begin{array}{lr}\text { A Equalizadores MMSE e ZF } & \mathbf{1 1 5}\end{array}$

$\begin{array}{lll}\text { A.1 Equalizador MMSE } & 115\end{array}$

A.1.1 Exemplo: OFDM-ZP 115

A.1.2 Exemplo: OFDM-CP 116

$\begin{array}{ll}\text { A.2 Equalizador ZF } & 117\end{array}$

$\begin{array}{ll}\text { A.2.1 Exemplo: OFDM-ZP } & 118\end{array}$

A.2.2 Exemplo: OFDM-CP 119 


\section{Lista de figuras}

2.1 Modelo do Transmissor 19

2.2 Equivalente passa-baixa do sistema de comunicações 23

2.3 Modelo vetorial de sinais 25

3.1 Modelo vetorial de sinais: OFDM-CP 28

3.2 Estrutura do Receptor Convencional OFDM-CP 30

3.3 Modelo vetorial de sinais: OFDM-ZP 31

3.4 Estrutura do Receptor Zero Forcing OFDM-ZP 32

3.5 Estrutura do Receptor MMSE OFDM-ZP 33

3.6 Estrutura do Receptor Zero Forcing Pós-DFT OFDM-ZP 35

4.1 Resposta em Freqüência do canal fixo 60

4.2 Erro quadrático médio, 'corrmatch', OFDM-ZP, $\frac{E_{B}}{N_{0}}=0 d B$

4.3 Erro quadrático médio, 'corrmatch', OFDM-ZP, $\frac{E_{B}}{N_{0}}=5 d B$

4.4 Erro quadrático médio, 'corrmatch', OFDM-ZP, $\frac{E_{B}}{N_{0}}=10 d B$

4.5 Erro quadrático médio, 'corrmatch', OFDM-ZP, $\frac{E_{B}}{N_{0}}=15 d B$

4.6 Erro quadrático médio, 'corrmatch', OFDM-ZP, $\frac{E_{B}}{N_{0}}=20 d B$

4.7 Erro quadrático médio, 'corrmatch', OFDM-ZP, $\frac{E_{B}}{N_{0}}=25 d B$

4.8 Erro quadrático médio, 'corrmatch', OFDM-ZP

4.9 Taxa de Erro de Bit, 'corrmatch', OFDM-ZP 65

4.10 Erro quadrático médio, 'Pot', OFDM-ZP, $\frac{E_{B}}{N_{0}}=0 d B$

4.11 Erro quadrático médio, 'Pot', OFDM-ZP, $\frac{E_{B}}{N_{0}}=5 d B$

4.12 Erro quadrático médio, 'Pot', OFDM-ZP, $\frac{E_{B}}{N_{0}}=10 d B$

4.13 Erro quadrático médio, 'Pot', OFDM-ZP, $\frac{E_{B}}{N_{0}}=15 d B$

4.14 Erro quadrático médio, 'Pot', OFDM-ZP, $\frac{E_{B}}{N_{0}}=20 d B$

4.15 Erro quadrático médio, 'Pot', OFDM-ZP, $\frac{E_{B}}{N_{0}}=25 d B$

4.16 Erro quadrático médio, 'Pot', OFDM-ZP

4.17 Taxa de Erro de Bit, 'Pot', OFDM-ZP 71

4.18 Resposta em Freqüência do canal fixo 72

4.19 Erro quadrático médio, 'corrmatch', OFDM-CP, $\frac{E_{B}}{N_{0}}=5 d B$

4.20 Erro quadrático médio, 'corrmatch', OFDM-CP, $\frac{E_{B}}{N_{0}}=15 d B$

4.21 Erro quadrático médio, 'corrmatch', OFDM-CP, $\frac{E_{B}}{N_{0}}=25 d B \quad 74$

4.22 Erro quadrático médio, 'corrmatch', OFDM-CP ${ }^{N_{0}} \quad 75$

4.23 Taxa de Erro de Bit, 'corrmatch', OFDM-CP 76

4.24 Erro quadrático médio, 'Pot', OFDM-CP, $\frac{E_{B}}{N_{0}}=5 d B$

4.25 Erro quadrático médio, 'Pot', OFDM-CP, $\frac{E_{B}}{N_{0}}=15 d B$

4.26 Erro quadrático médio, 'Pot', OFDM-CP, $\frac{E_{B}}{N_{0}}=25 d B$

4.27 Erro quadrático médio, 'Pot', OFDM-CP ${ }^{N_{0}} 78$

4.28 Taxa de Erro de Bit, 'Pot', OFDM-CP 79

4.29 Estimação de $\sigma^{2}$, 'corrmatch', OFDM-ZP, , $\hat{L}=L \quad 80$

4.30 Estimação de $\sigma^{2}$, 'corrmatch', OFDM-ZP, , $\hat{L}=D$

4.31 Resposta em Freqüência do canal fixo 82

4.32 Erro quadrático médio, Analítico, 'corrmatch', OFDM-ZP 83 
4.33 Resolvendo a ambigüidade, MSE

4.34 Resolvendo a ambigüidade, Taxa de Erro de Bit 87

5.1 Resposta ao Impulso do canal fixo 100

5.2 Resposta em Freqüência do canal fixo 101

5.3 Desempenho MSE, 'corrmatch-IBI', OFDM-ZP 101

5.4 Taxa de Erro de Bit, corrmatch-IBI, OFDM-ZP 102 


\section{Lista de tabelas}

5.1 Coeficientes do Canal

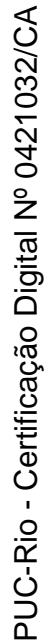

